\title{
CrystEngComm
}

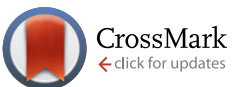

Cite this: CrystEngComm, 2015, 17, 4293

Received 23rd March 2015, Accepted 5th May 2015

DOI: $10.1039 / \mathrm{c} 5 \mathrm{ce} 00586 \mathrm{~h}$

www.rsc.org/crystengcomm

\section{Interplay of ligand chirality and metal configuration in mononuclear complexes and in a coordination polymer of $\mathrm{Cr}(\mathrm{III}) \dagger$}

\begin{abstract}
Ai Wang, Carina Merkens and Ulli Englert*
In order to investigate the influence of ligand chirality on the configuration of the coordinated metal, five pseudooctahedral $\mathrm{Cr}(\mathrm{III})$ complexes with one or two chelating $R, R$-1,2-diaminocyclohexane ligands have been synthesized. The mononuclear complexes $\left[\mathrm{Cr}(R, R-\mathrm{chxn})_{2} \mathrm{Cl}(\mathrm{DMSO})\right] \mathrm{Cl}_{2},\left[\mathrm{Cr}\left(R, R-\mathrm{Chxn}_{2} \mathrm{Cl}_{2}\right] \mathrm{Cl}_{\text {, }}\right.$ $\left[\mathrm{Cr}(\operatorname{acacCN})(R, R-\mathrm{chxn})_{2}\right]\left(\mathrm{NO}_{3}\right)_{2},\left[\mathrm{Cr}(\operatorname{acacCN})_{2}(R, R-\mathrm{chxn})\right] \mathrm{NO}_{3},\left[\mathrm{Cr}(\operatorname{acacCN})_{2}(R, R-\operatorname{chxn})\right] \mathrm{PF} 6 .(R, R-\operatorname{chxn}=R, R-$ 1,2-diaminocyclohexane; acacCN = deprotonated 3-cyanoacetylacetone and DMSO = dimethyl sulfoxide) have been obtained as crystalline solids, mostly solvates, and the potential chirality transfer from the enantiopure ligand to the configuration at the $\mathrm{Cr}(\mathrm{III})$ center has been investigated. The cationic complex $\left[\mathrm{Cr}(\operatorname{acacCN})_{2}(R, R \text {-chxn) }]^{+}\right.$has been synthesized as exclusively $\Lambda$ configured at the metal. In this complex, the dangling nitrile groups of the ditopic acacCN ligands may coordinate to $\mathrm{Ag}\left({ }^{\prime}\right)$ : the chiral-at-metal building block has thus been converted to the $2 \mathrm{D}$ network $\mathrm{Ag}\left[\mathrm{Cr}(\operatorname{acacCN})_{2}(R, R \text {-chxn) }]_{2}\left(\mathrm{PF}_{6}\right)_{3}\right.$ under retention of the stereochemistry at $\mathrm{Cr}(\mathrm{III})$. With respect to topology, the polycations in this mixed-metal coordination polymer correspond to two interpenetrated $\{4,4\}$ nets.
\end{abstract}

\section{Introduction}

An object not superimposable with its mirror image is called chiral. ${ }^{1}$ Chiral molecules are ubiquitous in living organisms, but chirality is not restricted to biomolecules: chiral materials and coordination compounds which may be obtained in enantiomerically pure form offer attractive potential, e.g. in asymmetric catalysis. Alfred Werner, ${ }^{2}$ the father of coordination theory, could prove as early as 1907 with the successful resolution of hexol that chirality is not necessarily associated with carbon. ${ }^{3}$ Nowadays, it is common knowledge that pseudo-octahedral complexes between a metal cation and three bidentate chelating ligands exist as $\Delta$ and $\Lambda$ isomers.

The potentially ditopic molecule 3-cyanoacetylacetone $(\mathrm{HacacCN})^{4}$ has been used for crystal engineering purposes. It may be deprotonated to a formally anionic ligand, combining the chelating pentanedionato moiety with an additional $\mathrm{N}$ coordination site; it can thus act as a linker in mixed-metal

Institute of Inorganic Chemistry, RWTH Aachen University, Aachen, Germany. E-mail: ullrich.englert@ac.rwth-aachen.de; Fax: +49 2418092 288;

Tel: +492418094666

$\dagger$ Electronic supplementary information (ESI) available: Crystallographic information in CIF format; details concerning crystallization, structure solution and refinement of 1-4 and 6-8; hydrogen bonds in 1, 2, 6 and 8; displacement ellipsoid plots of asymmetric unit for 1-4 and 6-8; powder patterns for 1, 2, 4, 5, 6, 7 and 8. CCDC 1047808-1047814. For ESI and crystallographic data in CIF or other electronic format see DOI: 10.1039/c5ce00586h coordination polymers. ${ }^{5-9} \mathrm{Cr}(\mathrm{III})$ represents a promising candidate for combining metal chirality with crystal engineering, for example, for the construction of chiral extended structures. It is a hard and inert cation for which only slow racemization can be expected. Its acetylacetonate complex has been successfully resolved into enantiomers ${ }^{10}$ by using a chiral base as deprotonating agent, followed by recrystallization. We can confirm this earlier report, but our attempts to resolve $\mathrm{Cr}(\mathrm{acacCN})_{3}$ into enantiomers in an analogous way were not successful: we only achieved low enantiomeric excess and very modest yields. An alternative way to induce a well-defined configuration about a metal cation relies on the use of organic ligands and transfer of the chiral information to the metal center. ${ }^{11-13}$ The combination of chirality at the metal with an enantiomerically pure ligand results in diastereomers which differ in their physical properties; under favourable conditions, the diastereomer of lower energy may then become the dominant reaction product. We have focused on the combination of $\Delta / \Lambda$ metal chirality in tris(chelating) pseudooctahedral complexes and $R R$ configuration in the chelating ligand $R, R-1,2$-diaminocyclohexane $(R$, $R$-chxn). The energetic preference for the $\Delta$ configuration at the central atom of the octahedral $\left[\mathrm{M}(R, R \text {-chxn })_{3}\right]^{3+}$ complex cations was established for $\mathbf{M}=\mathbf{C o}$ by Harnung and coworkers ${ }^{14}$ and is reflected by the fact that this diastereomer represents the readily available main product from crystallization experiments for $\left[\mathrm{M}(R, R \text {-chxn })_{3}\right] \mathrm{Cl}_{3} \cdot n \mathrm{H}_{2} \mathrm{O}(\mathrm{M}=\mathrm{Co}, \mathrm{Rh}),{ }^{15}$ $\left[\mathrm{Cr}(R, R-\operatorname{chxn})_{3}\right] \mathrm{Cl}_{3} \cdot n \mathrm{H}_{2} \mathrm{O}$ (ref. 16) and $\left[\mathrm{M}(R, R-\operatorname{chxn})_{3}\right] \mathrm{NO}_{3} \cdot n \mathrm{H}_{2} \mathrm{O}{ }^{17}$ 
Stoeckli-Evans and coworkers have successfully used enantiopure 1,2-diaminocyclohexane as chirality-inducing constituent in bimetallic assemblies. ${ }^{18,19}$ The same enantiopure diamine has been employed in the crystallization-induced resolution of biaryl derivatives. ${ }^{20}$ We shortly mention that not only enantiopure but also racemic 1,2-diaminocyclohexane has been applied in the field of crystal engineering: chxn complexes of transition metals have proven suitable counter cations for thioantimonates ${ }^{21}$ and indiumsulfates. ${ }^{22}$ As for the enantiopure diaminocyclohexane ligand, we hoped to exploit its central chirality and generate complexes of welldefined metal configuration containing only two or even only one molecule of $R, R$-chxn as a chelating ligand. The cations reported here adopt the composition $\left[\operatorname{Cr}(R, R-\operatorname{chxn})_{2} \mathrm{~L}_{2}\right]^{n+}$ (L = monodentate ligand, $n=1-2),\left[\mathrm{Cr}(\operatorname{acacCN})(R, R-\mathrm{chxn})_{2}\right]^{2+}$ or $\left[\mathrm{Cr}(\operatorname{acacCN})_{2}(R, R \text {-chxn })\right]^{+}$. Our special focus was on the latter complex for an obvious reason: a configurationally welldefined $\left[\mathrm{Cr}(\mathrm{acacCN})_{2}(R, R \text {-chxn })\right]^{+}$cation would represent a chiral-at-metal building block with two additional potential donor sites in the periphery and therefore be suitable for crosslinking to extended structures. The compounds investigated in this context have been summarized in Fig. 1.

\section{Results and discussion}

We will first address salts in which the cationic $\mathrm{Cr}$ (III) complex contains two chelating chxn and two additional monodentate ligands in the coordination sphere. $[\operatorname{Cr}(R, R-$ chxn $\left.)_{2} \mathrm{Cl}(\mathrm{DMSO})\right] \mathrm{Cl}_{2}$ and $\left[\mathrm{Cr}(R, R-\operatorname{chxn})_{2} \mathrm{Cl}_{2}\right] \mathrm{Cl}$ were synthesized by Pedersen ${ }^{23}$ in 1970, but their crystal structures have not yet been reported. We now provide this information: single crystals suitable for diffraction experiments of both compounds could be grown as DMSO solvate of the former (1) and EtOH hemisolvate of the latter (2) compound (Fig. 1); powder diffraction on the bulk (Fig. S16 and S17, ESI $\dagger$ ) confirmed that in either case a phase pure product was obtained. Like all solids reported here, these products are derivatives of enantiomerically pure $R, R$-chxn and hence necessarily crystallize in chiral space groups. Both 1 and 2 contain two complex cations per asymmetric unit but differ with respect to the stereochemistry at the metal: the $\left[\mathrm{Cr}(R, R-\operatorname{chxn})_{2} \mathrm{Cl}(\mathrm{DMSO})\right]^{2+}$ cations in 1 are both $\Delta$ configured whereas 2 features one $\left[\mathrm{Cr}(R, R-\mathrm{chxn})_{2} \mathrm{Cl}_{2}\right]^{+}$in $\Delta$ and one in $\Lambda$ configuration. A comment concerning short contacts between discrete residues seems appropriate: in view of the potential donors and acceptors, the existence of conventional hydrogen bonds in 1 and 2 as well as in all other structures reported here is no surprise. In 1, only the uncoordinated $\mathrm{Cl}^{-}$and DMSO residues accept $\mathrm{N}-\mathrm{H}$ hydrogen bonds. In contrast to this situation, the hydrogen bonds in 2 cover a wider distance range and both uncoordinated and metal coordinated groups act as acceptors. In both solids 1 and 2 , classical hydrogen bonds give raise to 2D layer structures. A synopsis of all classical hydrogen bonds in 1, 2, 6 and 8 is provided in Table S1 (ESI $\dagger$ ).

Our next aim was to introduce the ditopic linker HacacCN into the $\mathrm{Cr}$ (III) complexes, possibly under control of the configuration at the metal. The reaction was achieved by precipitating the chloride ligands in 1 or 2 with $\mathrm{AgNO}_{3}$ in the

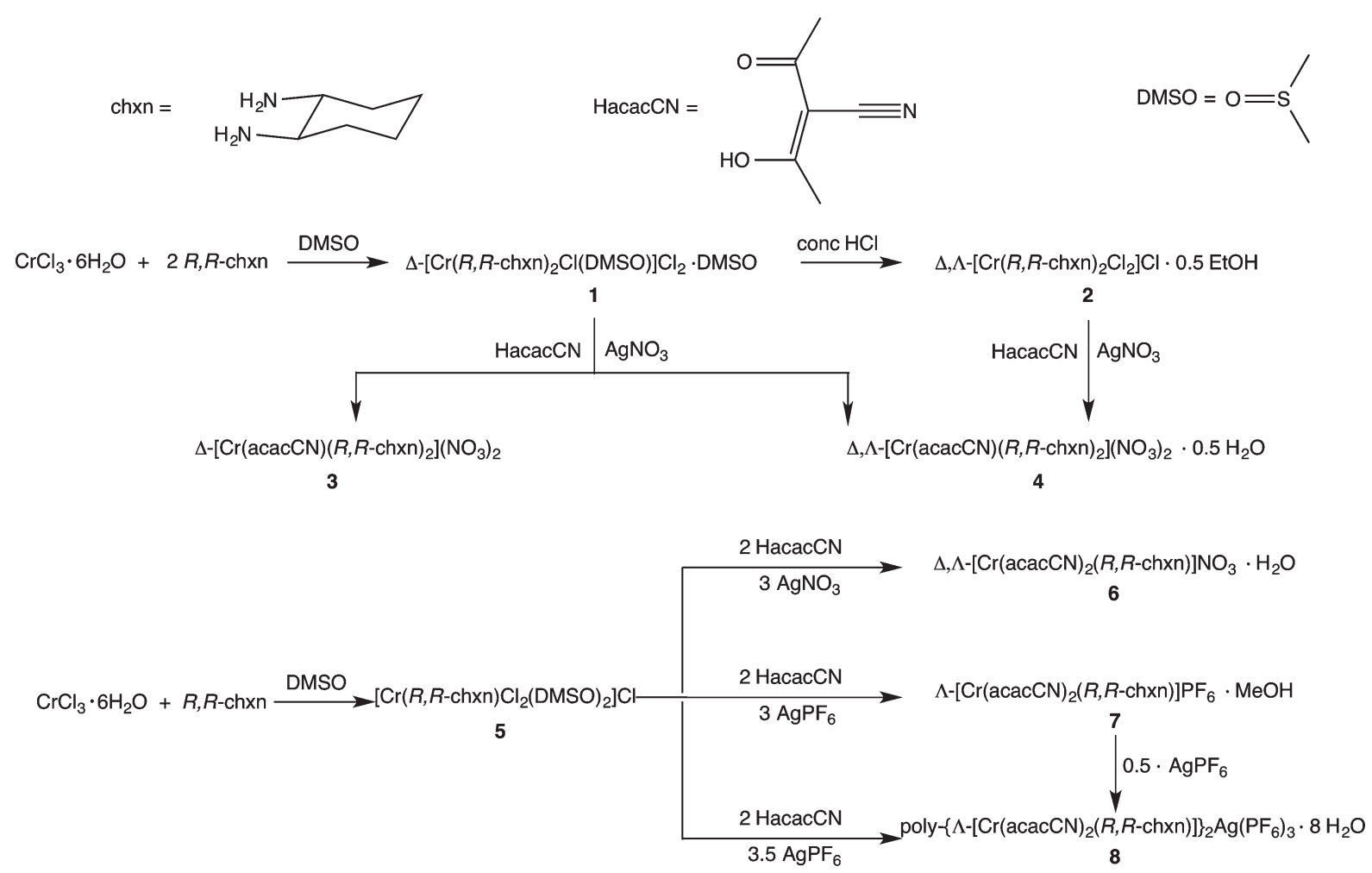

Fig. 1 Scheme of synthesis and naming convention for reactions and crystal structures determined in the context of this work. 
presence of the deprotonated acacCN ligand. The main reaction product is $\Delta, \Lambda$ - $\left[\mathrm{Cr}(\right.$ acacCN $\left.)(R, R \text {-chxn })_{2}\right]\left(\mathrm{NO}_{3}\right)_{2}$; it can be isolated as hemihydrate 4 . When the DMSO complex 1 is used as starting material, a second compound 3, $\Delta-\left[\mathrm{Cr}(\operatorname{acacCN})(R, R-\operatorname{chxn})_{2}\right]\left(\mathrm{NO}_{3}\right)_{2}$, is obtained concomitantly. We have been able to elucidate the stereochemistry at the $\mathrm{Cr}(\mathrm{III})$ centers in $\mathbf{3}$ and $\mathbf{4}$ by single crystal X-ray diffraction: 3 only contains $\Delta$ configured cations whereas 4 features complex cations in either configuration. The quantitative relationship between 3 and 4 has been established by powder diffraction of the bulk: Fig. 2 shows that reaction of 2 gives 4 as a phase pure solid, whereas the conversion of 1 results in a mixture in which 3 is present in only small quantities as a by-product. Unfortunately, the main product 4 does not meet our requirements for a single configuration at the metal.

4 and its by-product 3 are closely related with respect to packing. Fig. 3 shows projections of both structures along the shortest crystallographic axis. In 3, classical hydrogen bonds result in layers extending along the diagonal and the short axis - the hydrogen-bonded network is two-dimensional. In 4 , these layers are crosslinked by the additional water molecules in the crystal lattice (highlighted in Fig. 3 bottom); a 3D network of hydrogen bonds is formed. The presence of acacCN in the coordination sphere around $\mathrm{Cr}$ in 3 and 4 has another consequence for intermolecular interactions. The peripheral $\mathrm{CN}$ groups of the acacCN ligand represent additional potential acceptors for hydrogen bonds; such contacts also occur in 7. Table 1 summarises all classical hydrogen bonds in 3, 4 and 7; contacts with nitril acceptors have been highlighted in bold. The table shows that $\mathrm{CN} \cdots \mathrm{H}-\mathrm{N}$ bonds range between 3.0 and $3.1 \AA$ A. The only $\mathrm{CN} \cdots \mathrm{H}-\mathrm{O}$ interaction is due to a contact with the hydroxyl group of a cocrystallised $\mathrm{MeOH}$ molecule in 7 and is significantly shorter.

With respect to the stereochemistry at the $\mathrm{Cr}$ center, we can summarize our results for 1-4: each $\mathrm{Cr}(\mathrm{III})$ in these salts is coordinated to two enantiopure diaminocyclohexane ligands. The cationic $\mathrm{Cr}$ complexes either occur in $\Delta$ or as a mixture of $\Delta$ and $\Lambda$ configuration - no solid with a preference for a $\Lambda$

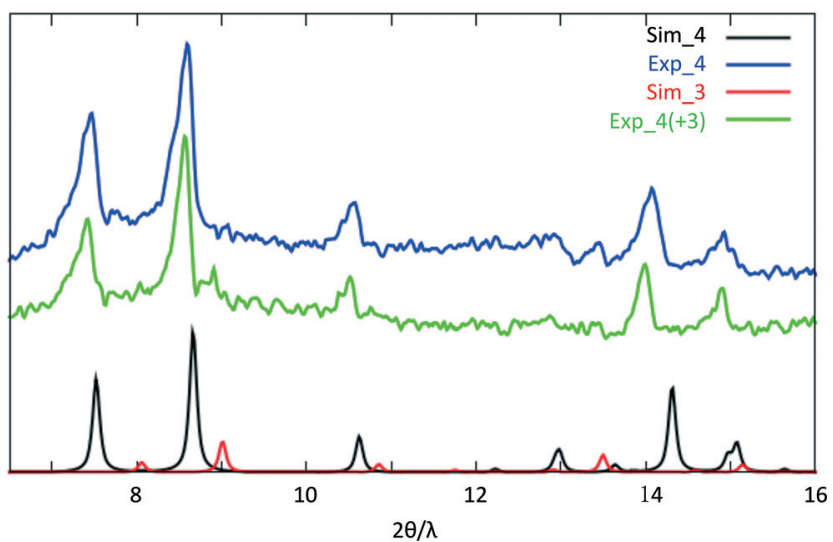

Fig. 2 The powder diffraction pattern of experimental and simulated of 3 and 4 .

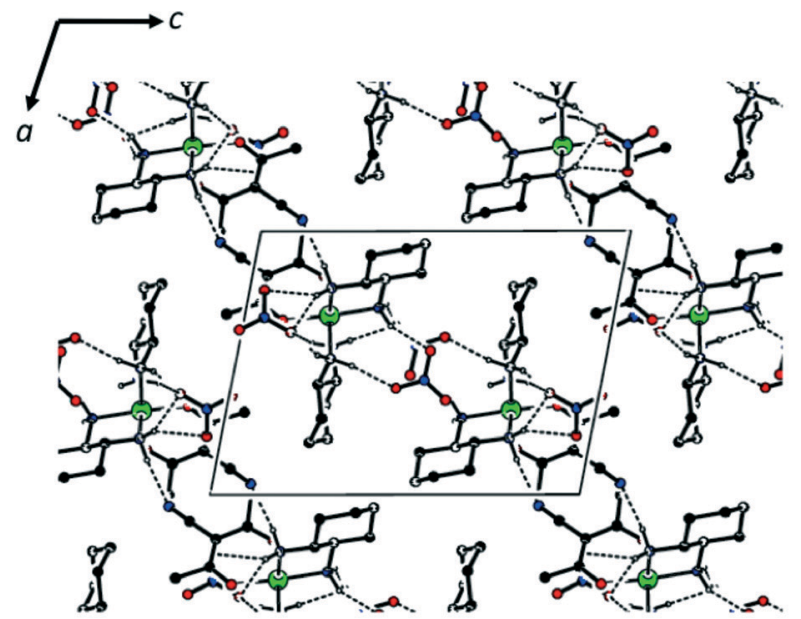

3

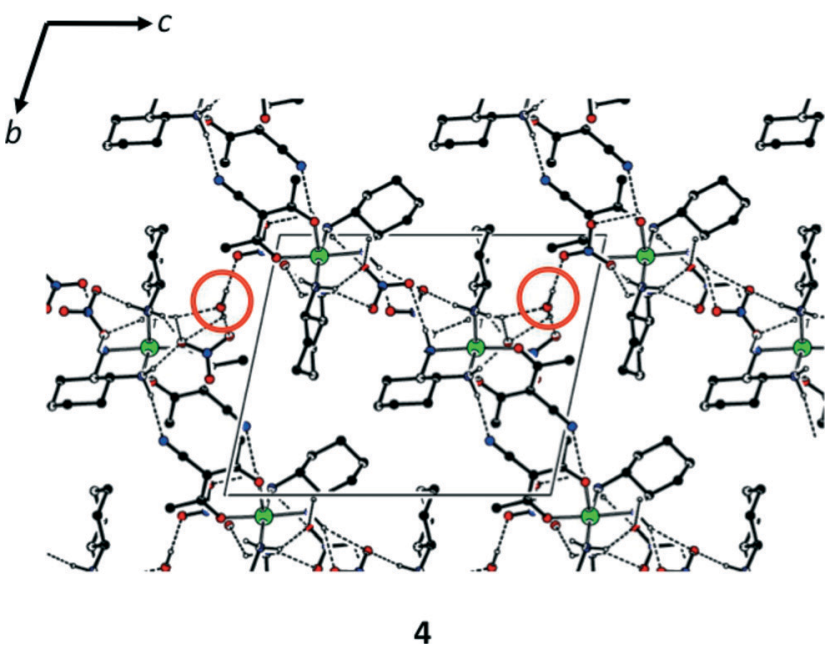

Fig. 3 Hydrogen bonds form layers in 3 (top) which are crosslinked by cocrystallized water molecules in 4 (bottom); the crosslinking water molecules have been highlighted by red circles.

configured cation has been encountered. However: in the crystalline products 2 and $4, \Delta$ and $\Lambda$ configuration coexist in $1: 1$ stoichiometry, and the concomitantly formed products 3 and 4 do not exhibit the same stereochemistry. These findings suggest that the energetic difference between the diastereomers $\Delta$-metal $/ R R$-chxn and $\Lambda$-metal $/ R R$-chxn is not very pronounced: other structural features such as the nature of the counteranions or the degree of solvation may well be decisive.

From a crystal engineering point of view, 3 and 4 represent dead ends because they offer only one terminal CN function for further coordination. At least two potential linker sites should be available for the construction of an extended solid. We therefore proceeded with the synthesis of the more suitable precursor $\left[\mathrm{Cr}(R, R\right.$-chxn $\left.) \mathrm{Cl}_{2}(\mathrm{DMSO})_{2}\right] \mathrm{Cl}, 5$, which we expected to convert into a building block with two ditopic acacCN ligands. This intermediate can be obtained in reasonable yield and may be characterised by elemental analysis. We have not been able to grow sufficiently large crystals for a full structural study of this compound, but the solubility 
Table 1 Classical hydrogen bonds ( $\AA$, ${ }^{\circ}$ ) of 3, 4 and 7, contacts involving peripheral $C N$ acceptors have been highlighted in bold

\begin{tabular}{|c|c|c|c|c|c|c|}
\hline Comp. & $\mathrm{D}-\mathrm{H} \cdots \mathrm{A}$ & D-H & $\mathrm{H} \cdots \mathrm{A}$ & $\mathrm{D} \cdots \mathrm{A}$ & $\angle \mathrm{D}-\mathrm{H} \cdots \mathrm{A}$ & Symmetry-for-A \\
\hline \multirow[t]{9}{*}{3} & N1-H1D $\cdots$ O8A & $0.98(5)$ & $2.06(5)$ & $2.975(11)$ & $154(4)$ & $1-x,-1 / 2+y, 1-z$ \\
\hline & N1-H1E $\cdots$ O6 & $0.98(3)$ & $2.02(3)$ & $2.894(7)$ & $147(5)$ & \\
\hline & N2-H2D $\cdots$ N5 & $0.98(4)$ & $2.14(4)$ & $3.096(7)$ & $165(5)$ & $-x,-1 / 2+y,-z$ \\
\hline & $\mathrm{N} 2-\mathrm{H} 2 \mathrm{E} \cdots \mathrm{O} 4$ & $0.99(5)$ & $2.16(5)$ & $3.042(6)$ & $148(4)$ & $-x,-1 / 2+y,-z$ \\
\hline & $\mathrm{N} 2-\mathrm{H} 2 \mathrm{E} \cdots \mathrm{O} 5$ & $0.99(5)$ & $2.25(5)$ & $2.999(6)$ & $131(4)$ & $-x,-1 / 2+y,-z$ \\
\hline & N3-H3D $\cdots \mathrm{O} 4$ & $0.99(4)$ & $2.00(5)$ & $2.868(6)$ & $146(4)$ & $-x, 1 / 2+y,-z$ \\
\hline & N3-H3E*O6 & $0.99(5)$ & $2.03(5)$ & $2.943(6)$ & $153(4)$ & \\
\hline & N4-H4D $\cdots$ O7A & $0.99(3)$ & $2.14(3)$ & $2.993(10)$ & $144(5)$ & $1-x,-1 / 2+y, 1-z$ \\
\hline & $\mathrm{N} 4-\mathrm{H} 4 \mathrm{E} \cdots \mathrm{O} 4$ & $0.98(4)$ & $2.13(5)$ & $3.065(6)$ & $158(6)$ & $-x,-1 / 2+y,-z$ \\
\hline \multirow[t]{22}{*}{4} & N1-H1D $\cdots \mathrm{O} 12$ & $0.98(3)$ & $2.44(4)$ & $3.329(8)$ & $151(4)$ & \\
\hline & N1-H1E $\cdots$ O6 & $0.98(4)$ & $2.05(4)$ & $2.966(7)$ & $156(4)$ & \\
\hline & $\mathrm{N} 2-\mathrm{H} 2 \mathrm{D} \cdots \mathrm{O} 6$ & $0.98(3)$ & $2.32(5)$ & $2.897(7)$ & $117(4)$ & $-1+x, y, z$ \\
\hline & $\mathrm{N} 2-\mathrm{H} 2 \mathrm{D} \cdots \mathrm{O} 17$ & $0.98(3)$ & $2.10(3)$ & $3.007(9)$ & $153(5)$ & $-1+x, y, z$ \\
\hline & $\mathrm{N} 2-\mathrm{H} 2 \mathrm{E} \cdots \mathrm{O} 11$ & $0.98(5)$ & $2.00(5)$ & $2.901(8)$ & $153(4)$ & $-1+x, y, z$ \\
\hline & N3-H3D $\cdots$ O16A & $0.99(3)$ & $2.23(3)$ & $3.204(10)$ & $168(4)$ & \\
\hline & N3-H3E $\cdots$ O11 & $0.99(4)$ & $2.01(4)$ & $2.863(7)$ & $143(4)$ & $-1+x, y, z$ \\
\hline & $\mathrm{N} 4-\mathrm{H} 4 \mathrm{D} \cdots \mathrm{N} 10$ & $0.99(3)$ & $2.14(3)$ & $3.124(7)$ & $170(4)$ & $x, 1+y, 1+z$ \\
\hline & $\mathrm{N} 4-\mathrm{H} 4 \mathrm{E} \cdots \mathrm{O} 6$ & $0.98(4)$ & $2.03(5)$ & $2.962(7)$ & $157(4)$ & \\
\hline & N6-H6D $\cdots O 9$ & $0.98(3)$ & $2.37(4)$ & $3.016(7)$ & $123(3)$ & $-1+x, y, z$ \\
\hline & N6-H6E $\cdots \mathrm{O} 14 \mathrm{~A}$ & $0.97(4)$ & $2.12(3)$ & $3.015(10)$ & $153(3)$ & \\
\hline & N7-H7D $\cdots$ O15A & $0.98(3)$ & $2.13(4)$ & $2.965(9)$ & $142(4)$ & $1+x, y, z$ \\
\hline & N7-H7E $\cdots O 9$ & $0.98(4)$ & $1.91(4)$ & $2.863(7)$ & $163(3)$ & \\
\hline & N8-H8D $\cdots$ O15A & $0.98(4)$ & $2.56(4)$ & $3.076(9)$ & $113(3)$ & $1+x, y, z$ \\
\hline & N8-H8E $\cdots \mathrm{O} 12$ & $0.97(3)$ & $2.36(3)$ & $3.266(7)$ & $154(4)$ & \\
\hline & N8-H8E $\cdots$ O13 & $0.97(3)$ & $2.07(4)$ & $2.939(6)$ & $148(3)$ & \\
\hline & N9-H9D $\cdots$ O15A & $0.97(4)$ & $2.44(4)$ & $3.327(9)$ & $152(3)$ & \\
\hline & N9-H9E $\cdots \mathrm{O} 10$ & $0.99(3)$ & $2.35(4)$ & $3.067(7)$ & $128(3)$ & $-1+x, y, z$ \\
\hline & N9-H9E $\cdots$ N5 & $0.99(3)$ & $2.28(4)$ & $3.070(7)$ & $136(3)$ & $x,-1+y,-1+z$ \\
\hline & $\mathrm{O} 1-\mathrm{H} 17 \cdots \mathrm{O} 6$ & 0.84 & 2.57 & $3.117(9)$ & 123 & \\
\hline & $\mathrm{O} 1-\mathrm{H} 17 \cdots \mathrm{O} 7$ & 0.84 & 1.79 & $2.632(12)$ & 180 & \\
\hline & $\mathrm{O} 1-\mathrm{H} 17 \cdots \mathrm{O} 8$ & 0.85 & 1.90 & $2.747(9)$ & 180 & $x, y, 1+z$ \\
\hline \multirow[t]{8}{*}{7} & N1-H1D $\cdots$ O5 & $0.98(3)$ & $2.02(3)$ & $2.966(5)$ & $163(4)$ & $1-x, 1 / 2+y, 1 / 2-z$ \\
\hline & $\mathrm{N} 1-\mathrm{H} 1 \mathrm{E} \cdots \mathrm{F} 1$ & $0.97(3)$ & $2.32(4)$ & $3.181(4)$ & $148(3)$ & $1-x, 1 / 2+y, 1 / 2-z$ \\
\hline & $\mathrm{N} 1-\mathrm{H} 1 \mathrm{E} \cdots \mathrm{F} 5$ & $0.97(3)$ & $2.10(2)$ & $2.986(4)$ & $151(3)$ & $1-x, 1 / 2+y, 1 / 2-z$ \\
\hline & $\mathrm{N} 2-\mathrm{H} 2 \mathrm{D} \cdots \mathrm{F} 3$ & $0.98(3)$ & $2.30(3)$ & $3.189(4)$ & $150(3)$ & $-1+x, y, z$ \\
\hline & $\mathrm{N} 2-\mathrm{H} 2 \mathrm{D} \cdots \mathrm{F} 4$ & $0.98(3)$ & $2.40(3)$ & $2.890(4)$ & $110(2)$ & $-1+x, y, z$ \\
\hline & $\mathrm{N} 2-\mathrm{H} 2 \mathrm{E} \cdots \mathrm{F} 4$ & $0.98(3)$ & $2.50(3)$ & $2.890(4)$ & $104(2)$ & $-1+x, y, z$ \\
\hline & $\mathbf{N} 2-\mathbf{H} 2 \mathbf{E}^{\cdots} \mathrm{N} 4$ & $0.98(3)$ & $2.06(2)$ & $3.020(5)$ & $163(3)$ & $-1 / 2+x, 1 / 2-y, 1-z$ \\
\hline & O5-H5O $\cdots$ N3 & $0.72(6)$ & $2.15(6)$ & $2.858(5)$ & $167(7)$ & $1 / 2+x, 1 / 2-y,-z$ \\
\hline
\end{tabular}

properties of $\mathbf{5}$ support its identity as a salt and its reactivity matches the expectation: with silver salts of weakly or noncoordinating anions, the precursor can be converted to salts containing tris(chelated) $\mathrm{Cr}(\mathrm{III})$ cations with two ditopic acacCN ligands in the coordination sphere. Repeated powder diffraction experiments showed good internal agreement and thus also confirmed that the synthesis of 5 leads to a reproducible product, albeit with unknown crystal structure.

Based on the intermediate 5, the nitrate 6 and the hexafluorophosphate 7 have been synthesised and fully characterised. Crystalline 6 is obtained as a rather stable and phase-pure hydrate; the result of the single crystal diffraction experiment matches the powder pattern of the bulk material (Fig. S20, ESI $\dagger$ ). The unit cell in space group $P 1$ contains four symmetrically independent cationic complexes, two in $\Delta$ and $\Lambda$. Fig. 4 shows that the overall shape of the cationic complex and the orientation of the chxn ligand in both isomers differ at first sight. As a consequence, packing properties and close contacts such as hydrogen bonds will also differ and affect the free enthalpy of any solid which contains these residues.
7 crystallises as a methanol solvate; larger single crystals may be isolated from the mother liquor and transferred into the cold $\mathrm{N}_{2}$ stream of the diffractometer whereas crystalline powder shows fast desolvation at room temperature (Fig. S21, ESI $\dagger$ ). When compared to the earlier reports concerning $\mathrm{M}(\mathrm{chxn})_{3}$ (ref. 14-17) and to the structural results for 1-4 discussed above, the stereochemistry at the metal in 7 is a

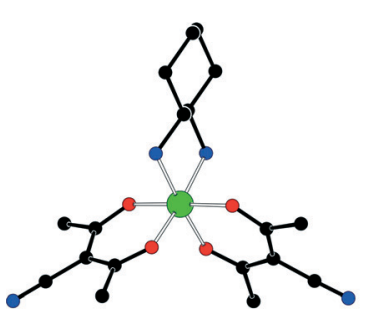

$\Delta$ configured cation in $\mathbf{6}$

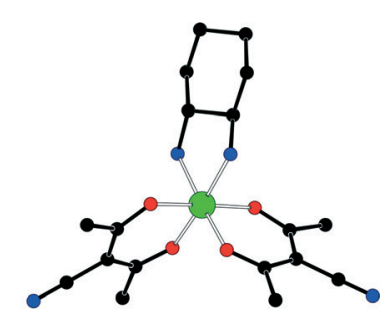

$\Lambda$ configured cation in 6
Fig. $4 \Delta$ (left) and $\Lambda$ (right) configured $\left[\mathrm{Cr}(\operatorname{acacCN})_{2}(R, R-\operatorname{ch} \times n)\right]^{+}$ cations in 6 . 
surprise: it represents the first solid in which only the $\Lambda$ configuration is encountered. The above mentioned preference for the $\Delta-R R$ configuration which was observed in $\left[\mathrm{M}(\mathrm{chxn})_{3}\right]^{3+}$ cations and in 1-4 with two chxn ligands per metal is obviously no longer valid if only one enantiopure chelating ligand coordinates with the chromium center as in our complexes 6 and 7. Despite this unexpected stereochemistry, the $\Lambda$-[Cr(acacCN $)_{2}(R, R$-chxn $\left.)\right]^{+}$cation in 7 matches our original requirements for crystal engineering: it represents a chiral-at-metal building block of defined configuration and allows extension via its two peripheral $\mathrm{N}$ donor sites. We recall that $\mathrm{Ag}(\mathrm{I})$ represents the preferred acceptor cation for this group; ${ }^{6,7} \mathrm{Ag}(\mathrm{I})$ was also used as a reagent to precipitate the halide ligands and generate vacant coordination sites at the $\mathrm{Cr}(\mathrm{III})$ precursor. Therefore a change in stoichiometry was sufficient to convert 5 into a polymer. Its hydrate 8 has been successfully studied by single crystal diffraction. Fig. 5 shows that the metal configuration has been retained and $\mathrm{Cr}(\mathrm{III})$ in 8 is exclusively $\Lambda$ configured, similar to the situation in 7 .

With a focus on crystal engineering, 8 represents the target solid of this study, constructed by bridging configurationally well-defined $\left[\mathrm{Cr}(\operatorname{acacCN})_{2}(R, R \text {-chxn })\right]^{+}$cations with $\mathrm{Ag}(\mathrm{I}) . \mathrm{PF}_{6}{ }^{-}$counter anions and hydrate water molecules occupy the voids in the resulting cationic framework. Each $\Lambda$-[Cr(acacCN $)_{2}(R, R$-chxn $\left.)\right]^{+}$building block is linked to two silver cations with its nitrile groups, and in agreement with the $\mathrm{Cr}: \mathrm{Ag}=2: 1$ stoichiometry, each $\mathrm{Ag}(\mathrm{I})$ is four-coordinated by cyano- $\mathrm{N}$ atoms of four different complex cations. The coordination sphere corresponds to an only slightly distorted tetrahedron, with $\mathrm{N}-\mathrm{Ag}-\mathrm{N}$ angles ranging between 99 and $123^{\circ}$. The program GTECS3 ${ }^{24,25}$ [GTECS3D is available free of charge for download from www.gtecs.rwth-aachen.de.] has been used to analyze the topology of the cationic framework in 8. When the $\left[\mathrm{Cr}(\text { acacaCN })_{2}(R, R\right.$-chxn $\left.)\right]$ complexes are taken as linkers and the $\operatorname{Ag}(\mathrm{I})$ cations as four-connected nodes, a $\{4,4\}$ net (Fig. 6, top) is obtained. Two such nets interpenetrate $^{26}$ in the crystal structure of 8 as becomes obvious after suitable simplification of the linkers (Fig. 6, bottom).

\section{Experimental section}

\subsection{Materials and methods}

Enantiopure (R,R-chxn) was obtained according to the method of Jacobsen et al. ${ }^{27}$ The HacacCN ligand was prepared

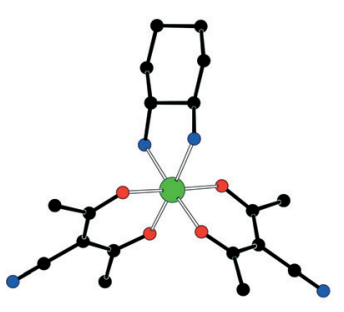

$\Lambda$ configured cation in 7

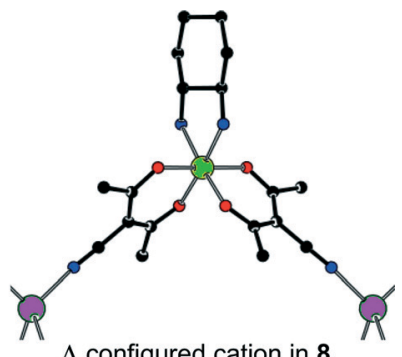

$\Lambda$ configured cation in $\mathbf{8}$
Fig. $5 \Lambda$ configuration in the discrete cations in 7 (left) and in the Agbridged cations of polymer 8 (right).

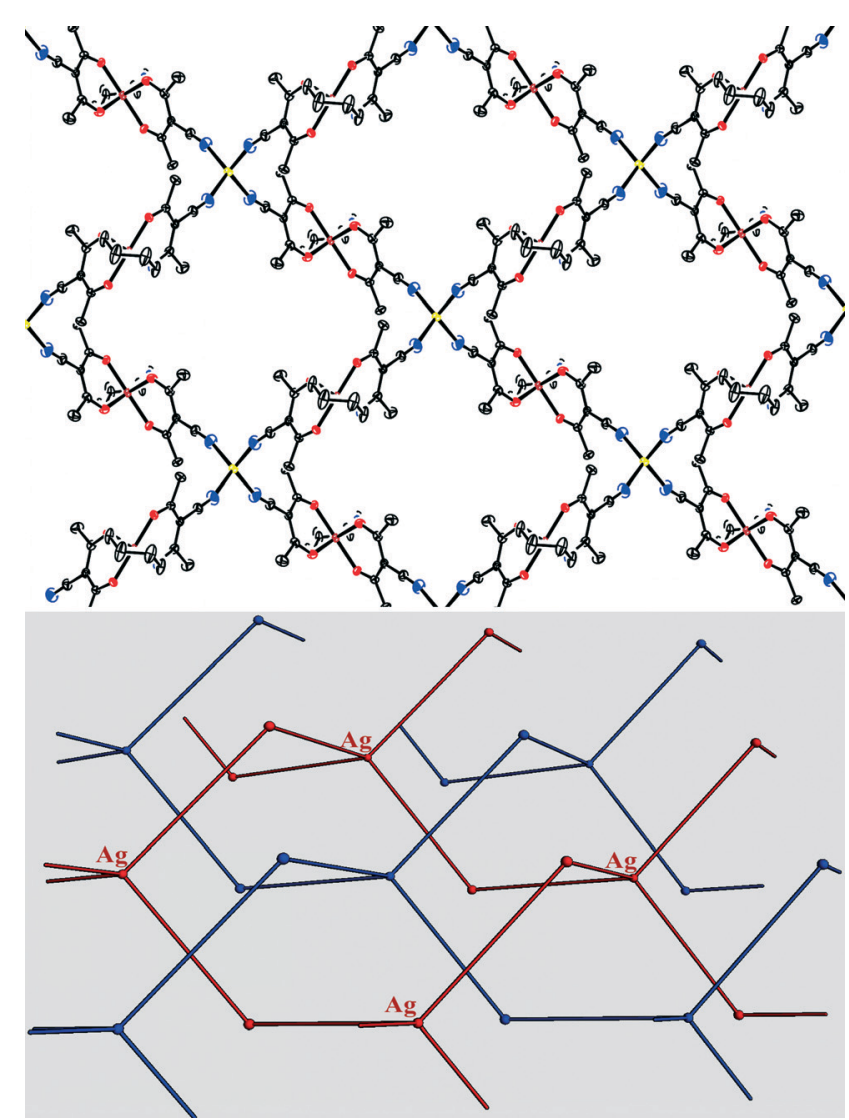

Fig. 6 Section of 8 : $\{4,4\}$ net formed by $\left[\mathrm{AgCr}(\operatorname{acacCN})_{2}(R, R-\mathrm{ch} \times n)\right]_{2}$ polycations (top); two such networks interpenetrate in the crystal structure of $\mathbf{8}$ (bottom).

according to the literature method by Silvernail et al. $;^{4}$ the final purification was made by sublimation. Complexes 1 and 2 were synthesized according to the procedure reported by Pedersen. ${ }^{23}$ Other chemicals were used without further purification: $\mathrm{CrCl}_{3} \cdot 6 \mathrm{H}_{2} \mathrm{O}$ (98\%, Grüssing), $\mathrm{NaHCO}_{3}(99.5 \%$, KMF Laborchemie), $\mathrm{AgPF}_{6}\left(98 \%\right.$, Aldrich) and $\mathrm{AgNO}_{3}$ (99.5\%, Fluka Chemie). IR spectra were recorded on a Nicolet Avatar 360 E.S.P. spectrometer in $\mathrm{KBr}$ pellets. In order to exclude artefacts by reaction of the silver-containing products with $\mathrm{KBr}$, comparisons with IR spectra in nujol mulls were made; they gave satisfactory agreement. CHN microanalyses were carried out at the Institute of Organic Chemistry, RWTH Aachen University, using a HERAEUS CHNO-Rapid. Powder diffraction experiments were performed at room temperature on flat samples with a Stoe \& Cie STADI P diffractometer equipped with an image plate detector with constant $\omega$ angle of $55^{\circ}$ using germanium-monochromated $\mathrm{Cu}-\mathrm{K} \alpha_{1}$ radiation $(\lambda=1.54051 \AA)$.

\subsection{Synthesis}

3.2.1 Synthesis of $\left\{\left[\operatorname{Cr}(\operatorname{acacCN})(R, R \text {-chxn })_{2}\right]\left(\mathrm{NO}_{3}\right)_{2}\right\}, 3$ and $\left\{\left[\mathrm{Cr}(\operatorname{acacCN})(R, R-\operatorname{chxn})_{2}\right]\left(\mathrm{NO}_{3}\right)_{2} \cdot 0.5 \mathrm{H}_{2} \mathrm{O}\right\}, 4.27 \mathrm{mg}(0.22 \mathrm{mmol})$ HacacCN and $18 \mathrm{mg}(0.22 \mathrm{mmol}) \mathrm{NaHCO}_{3}$ were completely dissolved in $15 \mathrm{ml} \mathrm{H}_{2} \mathrm{O} .100 \mathrm{mg}(0.22 \mathrm{mmol}) 1$ were added 
Table 2 Crystal data and refinement results of the 1, 2, 3 and 4

\begin{tabular}{|c|c|c|c|c|}
\hline Molecular weight & 464.87 & 815.20 & 528.19 & 1074.38 \\
\hline Cryst. system & Monoclinic & Monoclinic & Monoclinic & Triclinic \\
\hline Space group & $P 2_{1}$ & $C 2$ & $P 2_{1}$ & $P 1$ \\
\hline$a(\AA)$ & 11.9663(17) & $25.314(6)$ & $11.171(4)$ & $7.1767(5)$ \\
\hline$\beta\left({ }^{\circ}\right)$ & $99.533(2)$ & $111.163(5)$ & 101.113(5) & $93.4647(11)$ \\
\hline$\gamma\left({ }^{\circ}\right)$ & & & & $106.3580(11)$ \\
\hline$V\left(\AA^{3}\right)$ & $2703.2(7)$ & $3849.6(16)$ & $1224.3(7)$ & $1246.06(15)$ \\
\hline Total/unique reflections & $32572 / 11177$ & $16862 / 7551$ & $18562 / 7057$ & 19348/14 018 \\
\hline$R_{1}$ (all data) & 0.0468 & 0.1095 & 0.0712 & 0.0645 \\
\hline$R\left[F^{2}>2 \sigma\left(F^{2}\right)\right]$ & 0.0378 & 0.0676 & 0.0638 & 0.0538 \\
\hline Flack parameter & $0.00(2)$ & $0.08(4)$ & $0.03(3)$ & $0.01(2)$ \\
\hline CCDC & 1047808 & 1047809 & 1047810 & 1047811 \\
\hline
\end{tabular}

Table 3 Crystal data and refinement results of the 6,7 and 8

\begin{tabular}{|c|c|c|c|}
\hline Structure & 6 & 7 & 8 \\
\hline Molecular weight & 494.45 & 591.44 & 1516.02 \\
\hline Space group & $P 1$ & $P 2{ }_{1} 2_{1} 2_{1}$ & $C 222$ \\
\hline$a(\AA)$ & $9.5030(6)$ & $8.2870(17)$ & $10.078(2)$ \\
\hline$b(\AA)$ & $13.7410(9)$ & $14.806(3)$ & $42.812(9)$ \\
\hline$\beta\left({ }^{\circ}\right)$ & $83.8466(12)$ & & \\
\hline$\gamma\left({ }^{\circ}\right)$ & $71.8161(11)$ & & \\
\hline$V\left(\AA^{3}\right)$ & $2303.2(3)$ & 2621.7(9) & 3149.6(12) \\
\hline Total/unique reflections & $36098 / 26058$ & $36241 / 7216$ & $238881 / 4682$ \\
\hline$R_{1}$ (all data) & 0.0645 & 0.0577 & 0.0743 \\
\hline$R\left[F^{2}>2 \sigma\left(F^{2}\right)\right]$ & 0.0512 & 0.0451 & 0.0622 \\
\hline Flack parameter & $0.003(7)$ & $-0.002(14)$ & $0.018(14)$ \\
\hline CCDC & 1047812 & 1047813 & 1047814 \\
\hline
\end{tabular}

and the solution turned purple. $112 \mathrm{mg}$ (0.66 mmol) $\mathrm{AgNO}_{3}$ in $3 \mathrm{~mL}$ of water were added dropwise, and stirred for 16 hours at room temperature. The precipitate was removed by filtration, the clear solution was evaporated and the residue was dried in a desiccator. The raw product is an orange solid, which was purified by recrystallization from water. Yield: 120 $\mathrm{mg}$ (0.23 mmol, 53\%). Powder diffraction (see Fig. 2) shows that the reaction product mainly consists of 4 , with a small amount of 3 as byproduct.

3.2.2 Synthesis of $\left\{\left[\mathrm{Cr}(\operatorname{acacCN})(R, R-\operatorname{chxn})_{2}\right]\left(\mathrm{NO}_{3}\right)_{2} \cdot 0.5 \mathrm{H}_{2} \mathrm{O}\right\}$, 4. $16 \mathrm{mg}(0.13 \mathrm{mmol}) \mathrm{HacacCN}$ and $11 \mathrm{mg}(0.13 \mathrm{mmol})$ $\mathrm{NaHCO}_{3}$ were completely dissolved in $5 \mathrm{ml} \mathrm{H}_{2} \mathrm{O}$. Then
$50 \mathrm{mg}(0.13 \mathrm{mmol}) 2$ were added, and the solution turned purple. $66 \mathrm{mg}(0.39 \mathrm{mmol}) \mathrm{AgNO}_{3}$ in $5 \mathrm{~mL}$ of water were added dropwise, and stirred for 16 hours at room temperature. The precipitate was removed by filtration, the clear solution was evaporated under vacuum and the residue was dried in a desiccator. The raw product is an orange solid, which was purified by recrystallization from water. Yield: 31 $\mathrm{mg}$ (0.06 mmol, 47\%). The product systematically gives a too low carbon content in elemental analysis, but the powder pattern of the bulk matches the simulation based on the single crystal structure (ESI, $\dagger$ Fig. S7 and S8). IR: $v(\mathrm{C} \equiv \mathbf{N}$, $\left.\mathrm{cm}^{-1}\right)=2216$. 
3.2.3 Synthesis of $\left\{\left[\mathrm{Cr}(R, R-\operatorname{chxn}) \mathrm{Cl}_{2}(\mathrm{DMSO})_{2}\right](\mathrm{Cl})\right\}$, 5. The synthesis of 5 was carried out in analogy to the method by Pedersen, ${ }^{23}$ but with different stoichiometry. $2.333 \mathrm{~g} \mathrm{CrCl}_{3}$ $\cdot 6 \mathrm{HO}_{2}(8.76 \mathrm{mmol})$ were dissolved in $15 \mathrm{~mL}$ dimethylsulfoxide (DMSO) and heated to $180{ }^{\circ} \mathrm{C}$ to remove the water. After cooling to $110{ }^{\circ} \mathrm{C}, 1.000 \mathrm{~g} R, R$-chxn $(8.76 \mathrm{mmol})$ in 5 mL DMSO was added. A few minutes later, a silky red powder started to precipitate. The reaction was kept at $100{ }^{\circ} \mathrm{C}$ for 1 hour and then allowed to cool to room temperature; the product was precipitated with $150 \mathrm{~mL}$ EtOH. This crude product was filtered, washed with $\mathrm{EtOH}$ and dried in a desiccator. Yield: $1.941 \mathrm{~g}(4.53 \mathrm{mmol}, 52 \%)$. Anal. calcd for $5\left[\mathrm{C}_{10} \mathrm{H}_{26} \mathrm{Cl}_{3} \mathrm{CrN}_{2} \mathrm{O}_{2} \mathrm{~S}_{2}\right]: \mathrm{C}, 28.01 ; \mathrm{H}, 6.11 ; \mathrm{N}, 6.56$. Found: $\mathrm{C}$, 28.28; H, 6.15; N, 7.56.

3.2.4 Synthesis of $\left\{\left[\mathrm{Cr}(\operatorname{acacCN})_{2}(R, R-\operatorname{chxn})\right]\left(\mathrm{NO}_{3}\right) \cdot \mathrm{H}_{2} \mathrm{O}\right\}, 6$. $117 \mathrm{mg}$ (0.94 mmol) HacacCN and $78 \mathrm{mg}(0.94 \mathrm{mmol})$ $\mathrm{NaHCO}_{3}$ were added to $5 \mathrm{~mL}$ MeOH under stirring. After complete dissolution $200 \mathrm{mg}(0.47 \mathrm{mmol})$ of 5 in $5 \mathrm{~mL}$ of water were added. $238 \mathrm{mg}(1.41 \mathrm{mmol})$ of $\mathrm{AgNO}_{3}$ in $5 \mathrm{~mL}$ of water were added to the purple solution dropwise and stirred for 16 hours at room temperature. The precipitate was removed by decanting and filtration; the clear red solution was stored at room temperature and allowed to evaporate slowly. After four weeks, the raw product was obtained. Single crystal were grown by recrystallization from $\mathrm{MeOH}$ solvent. Yield: $102 \mathrm{mg}(0.21 \mathrm{mmol}, 44 \%)$. Anal. calcd for 6 $\left[\mathrm{C}_{18} \mathrm{H}_{28} \mathrm{CrN}_{5} \mathrm{O}_{8}\right]$ : C, 43.73; H, 5.71; N, 14.16. Found: C, 45.53; $\mathrm{H}, 5.74 ; \mathrm{N}, 13.73$. IR: $v\left(\mathrm{C} \equiv \mathrm{N}, \mathrm{cm}^{-1}\right)=2212$.

3.2.5 Synthesis of $\left\{\left[\mathrm{Cr}(\operatorname{acacCN})_{2}(R, R-\operatorname{chxn})\right]\left(\mathrm{PF}_{6}\right) \cdot \mathrm{MeOH}\right\}, 7$. $100 \mathrm{mg}(0.23 \mathrm{mmol})$ of 5 were dissolved in $5 \mathrm{~mL} \mathrm{MeOH}$ and stirred for $5 \mathrm{~min} .58 \mathrm{mg}(0.46 \mathrm{mmol})$ of HacacCN and $39 \mathrm{mg}$ ( $0.46 \mathrm{mmol})$ of $\mathrm{NaHCO}_{3}$ were completely dissolved in a mixture of $2 \mathrm{~mL}$ water and $5 \mathrm{~mL} \mathrm{MeOH}$. Both solutions were combined; after stirring for $20 \mathrm{~min}$, the reaction mixture turned purple. $177 \mathrm{mg}(0.70 \mathrm{mmol})$ of $\mathrm{AgPF}_{6}$ in $5 \mathrm{~mL} \mathrm{MeOH}$ were added dropwise. Stirring was continued at room temperature for $8 \mathrm{~h}$, and then the precipitate was separated by centrifugation. Even after prolonged reaction times, very small amounts of $\mathrm{AgCl}$ continued to precipitate and prevent crystallization of the target product; therefore, the clear solution was exposed for 18 hours to UV radiation $(\lambda=366 \mathrm{~nm})$ in order to remove traces of $\mathrm{Ag}$ (I) in solution. The black $\mathrm{Ag}$ thus formed was removed by filtration, and the clear red solution was allowed to evaporate at ambient temperature. Red blockshaped crystals formed after 3d. Yield: $44 \mathrm{mg}(0.08 \mathrm{mmol}$, $34 \%)$. Anal. calcd for 7 without $\mathrm{MeOH}\left[\mathrm{C}_{18} \mathrm{H}_{26} \mathrm{~F}_{6} \mathrm{CrN}_{4} \mathrm{O}_{4} \mathrm{P}\right]$ : C, 38.68; H, 4.68; N, 10.02. Found: C, 38.55; H, 4.65; N, 10.03 . IR: $v\left(\mathrm{C} \equiv \mathrm{N}, \mathrm{cm}^{-1}\right)=2212 ; v\left(\mathrm{P}-\mathrm{F}, \mathrm{cm}^{-1}\right)=722$.

3.2.6 Synthesis of $\left\{\Lambda-\left[\operatorname{Cr}(\operatorname{acacCN})_{2}(R, R-\operatorname{chxn})\right]_{2} \mathbf{A g}\left(\mathbf{P F}_{6}\right)_{3}\right.$ $\left..8 \mathrm{H}_{2} \mathrm{O}\right\}, 8.100 \mathrm{mg}(0.23 \mathrm{mmol})$ of 5 were dissolved in $5 \mathrm{~mL}$ $\mathrm{MeOH}$ and stirred for $5 \mathrm{~min} .58 \mathrm{mg}(0.46 \mathrm{mmol})$ of HacacCN and $39 \mathrm{mg}(0.46 \mathrm{mmol})$ of $\mathrm{NaHCO}_{3}$ were completely dissolved in a mixture of $2 \mathrm{~mL}$ water and $5 \mathrm{~mL} \mathrm{MeOH}$. Both solutions were combined; after stirring for $20 \mathrm{~min}$, the reaction mixture turned purple. $177 \mathrm{mg}(0.70 \mathrm{mmol})$ of $\mathrm{AgPF}_{6}$ in $5 \mathrm{~mL} \mathrm{MeOH}$ were added dropwise. Stirring was continued at room temperature for $8 \mathrm{~h}$, and then the precipitate was separated by centrifugation. As in the case of 7 , the solution was exposed for 18 hours to UV radiation $(\lambda=366 \mathrm{~nm})$ to remove traces of $\mathrm{Ag}(\mathrm{I})$, and the black $\mathrm{Ag}$ thus formed was removed by filtration. The clear red filtrate and a solution of $30 \mathrm{mg}$ (0.12 mmol) $\mathrm{AgPF}_{6}$ in $3 \mathrm{~mL} \mathrm{MeOH}$ were combined, and the resulting mixture was kept at room temperature in the dark. After 3d, a small quantity of red plate-shaped crystals were harvested. Yield: $35 \mathrm{mg}$ (0.05 mmol, 20\%). Anal. calcd for $8\left[\mathrm{C}_{18} \mathrm{H}_{34} \mathrm{Ag} 0.5 \mathrm{~F}_{9} \mathrm{CrN}_{4} \mathrm{O}_{8} \mathrm{P}_{1.5}\right]: \mathrm{C}, 28.53 ; \mathrm{H}, 4.52 ; \mathrm{N}, 7.39$. Found: C, 27.11; H, 3.80; N, 6.74. IR: $v\left(\mathrm{C} \equiv \mathrm{N}, \mathrm{cm}^{-1}\right)=2217$ with shoulder at $2231 ; v\left(\mathrm{P}-\mathrm{F}, \mathrm{cm}^{-1}\right)=722$.

\subsection{Crystallographic studies}

All intensity data were collected at $100 \mathrm{~K}$ on a Bruker D8 goniometer equipped with an APEX CCD area detector and an Incoatec microsource using Mo $K \alpha$ radiation $(\lambda=$ $0.71073 \mathrm{~A})$. Constant temperature was maintained by an Oxford Cryosystems 700 controller. The intensity data were integrated using the program SAINT+ (ref. 28) and corrected for absorption by multi-scan methods with SADABS. ${ }^{29}$ The structures were solved by direct methods (SHELXS-13) ${ }^{30}$ and refined by full matrix least square procedures based on $F^{2}$ (SHELXL-13). ${ }^{31}$ Non-hydrogen atoms were refined with anisotropic displacement parameters. Hydrogen atoms connected to carbon atoms were placed in idealized positions and included as riding. Tables 2 and 3 contain crystal data, data collection parameters and convergence results. Details about disorder treatment and refinement of $\mathrm{H}$ atoms bonded to hetero atoms have been compiled in the ESI. $\dagger$ The final structure models are available in CIF format, CCDC no. 10478081047811(1-4) and 1047812-1047814(6-8).

\section{Conclusions}

Starting from an enantiopure ligand such as $R, R-1,2-$ diaminocyclohexane, the synthesis of chiral solids is a trivial task. Transfer of ligand chirality to the configuration of the central cation is less obvious. Are we telling a success story? With respect to our initial focus, an affirmative answer can be given: making use of the chiral information from the enantiopure ligand, we have been able to synthesize a chiralat-metal cationic building block $\left[\mathrm{Cr}(\operatorname{acacCN})_{2}(R, R \text {-chxn })\right]^{+}$and a related coordination network. The stereochemistry in these target solids is consistent, in agreement with the idea that the configuration once achieved for such an inert complex should be maintained upon further crosslinking even if the resulting coordination polymer should not correspond to the thermodynamically most stable product. With respect to chirality transfer within a building block and crystal engineering of an extended solid, our approach was successful. And yet we encountered the unexpected: the configuration at the metal in $\left[\mathrm{Cr}(\operatorname{acacCN})_{2}(R, R \text {-chxn })\right]^{+}$cannot be predicted by simple extrapolation from $\left[\mathrm{Cr}(R, R \text {-chxn })_{3}\right]^{3+}$ via cationic $\left[\mathrm{Cr}(\text { acacCN })(R, R \text {-chxn })_{2}\right]^{2+}$ complexes. The enantiopure ligand and the alternative $\Delta$ and $\Lambda$ configurations at the center of 
coordination result in diastereomers. These diastereomers in principal differ in energy, but the synopsis of our structural results indicates that the associated energy differences are modest. The energy of a building block obviously does not represent the decisive quantity for the outcome of a crystallisation experiment: interionic Coulomb forces, hydrogen bonds and ubiquitous weaker interactions also contribute to the free enthalpy of the product solids and may well overcompensate the effect of a slightly less favourable diastereomeric complex cation. Fig. 4 shows that the alternative diastereomers differ in shape, charge distribution and orientation of potential hydrogen bond donors and acceptors. Future work will therefore be devoted to understand the role of the remaining constituents, in particular the counteranions engaged in the doubtlessly relevant electrostatic interactions. The direct comparison between the stereochemistry in 6 and 7 suggests that they may be decisive for the configuration at the $\mathrm{Cr}$ (III) cation.

\section{Acknowledgements}

Financial support by China Scholarship Council (A. W.) and RWTH Graduiertenförderung (C. M.) is gratefully acknowledged. The authors thank Irmgard Kalf and Paul Müller for help with synthesis and powder diffraction.

\section{References}

1 E. L. Eliel and S. H. Wilen, Stereochemistry of organic compounds, John Wiley \& Sons, New York, 1993.

2 E. B. Bauer, Chem. Soc. Rev., 2012, 41, 3153-3167.

3 E. Z. A. Werner, E. Berl and B. G. Jantsch, Ber. Dtsch. Chem. Ges., 1907, 40, 2103-2125.

4 C. Silvernail, G. Yap, R. Sommer, A. Rheingold, V. Day and J. Belot, Polyhedron, 2001, 20, 3113-3117.

5 A. D. Burrows, K. Cassar, M. F. Mahon and J. E. Warren, Dalton Trans., 2007, 2499-2509.

6 M. Kondracka and U. Englert, Inorg. Chem., 2008, 47, 10246-10257.

7 C. Merkens, N. Becker, K. Lamberts and U. Englert, Dalton Trans., 2012, 41, 8594-8599.

8 C. Merkens and U. Englert, Dalton Trans., 2012, 41, 4664-4673.

9 C. Merkens, O. Pecher, F. Steuber, S. Eisenhut, A. Görne, F. Haarmann and U. Englert, Z. Anorg. Allg. Chem., 2013, 639, 340-346.
10 S. F. Mason, R. D. Peacock and T. Prosperi, J. Chem. Soc., Dalton Trans., 1977, 702-704.

11 U. Knof and A. von Zelewsky, Angew. Chem., Int. Ed., 1999, 38, 302-322.

12 J. Crassous, Chem. Soc. Rev., 2009, 38, 830-845.

13 J. Crassous, Chem. Commun., 2012, 48, 9687-9695.

14 S. Harnung, B. Soendergaard Soerensen, I. Creaser, H. Maegaard, U. Pfenninger and C. Sächffer, Inorg. Chem., 1976, 15, 2123-2126.

15 M. Ito, F. Marumo and Y. Saito, Acta Crystallogr., Sect. B: Struct. Sci., 1971, 27, 2187-2195.

16 I. Kalf, B. Calmuschi and U. Englert, CrystEngComm, 2002, 4, 548-551.

17 M. Morooka, S. Ohba and H. Miyamae, Acta Crystallogr., Sect. B: Struct. Sci., 1992, 48, 667-672.

18 O. Sereda, A. Neels, F. Stoeckli and H. Stoeckli-Evans, Cryst. Growth Des., 2008, 8, 3380-3384.

19 O. Sereda, H. Stoeckli-Evans, O. Dolomanov, Y. Filinchuk and P. Pattison, Cryst. Growth Des., 2009, 9, 3168-3176.

20 H. Degenbeck, A.-S. Felten, J. Etxebarria, E. Escudero-Adan, J. Benet-Buchholz and A. Vidal-Ferran, Cryst. Growth Des., 2012, 12, 2719-2723.

21 L. Engelke, C. Näther, P. Leisner and W. Bensch, Z. Anorg. Allg. Chem., 2008, 634, 2959-2965.

22 Y.-H. Wang, J.-B. Jiang, P. Wang, X.-L. Sun, Q.-Y. Zhu and J. Dai, CrystEngComm, 2013, 15, 6040-6045.

23 E. Pedersen, Acta Chem. Scand., 1970, 24, 3362-3372.

24 K. Lamberts, S. Porsche, B. Hentschel, T. Kuhlen and U. Englert, CrystEngComm, 2014, 16, 3305-3311.

25 K. Lamberts, C. Merkens, R. Wang, U. Englert, D. Hons, S. Grüter, Y. Guo, S. Porsche, A. Hamacher, D. Bündgens and T. Kuhlen, Z. Anorg. Allg. Chem., 2012, 32, 117.

26 L. Carlucci, G. Ciani, D. M. Proserpio, T. G. Mitina and V. A. Blatov, Chem. Rev., 2014, 114, 7557-7580.

27 J. F. Larrow, E. N. Jacobsen, Y. Gao, Y. Hong, X. Nie and C. M. Zepp, J. Org. Chem., 1994, 59, 1939-1942.

28 SAINTt. (Version 7.68.), Program for reduction of data collected on bruker CCD area detector diffractometer, 2009.

29 G. M. Sheldrick, SADABS (version 2.03). A program for empirical absorption correction, University of Göttingen, Germany, 2004.

30 G. M. Sheldrick, Acta Crystallogr., Sect. A: Found. Crystallogr., 2008, 64, 112-122.

31 G. M. Sheldrick, Acta Crystallogr., Sect. C: Struct. Chem., $2015,71,3-8$. 